\title{
Pre-breakdown Arcing and Electrostatic Discharge in Dielectrics under High DC Electric Field Stress
}

\author{
Allen Andersen and JR Dennison \\ Materials Physics Group \\ Utah State University \\ 4415 Old Main Hill, Logan, UT 84322 USA
}

\begin{abstract}
Highly disordered insulating materials exposed to high electric fields will, over time, degrade and fail, potentially causing catastrophic damage to devices. Step-up to electrostatic discharge (ESD) tests were performed for two common polymer dielectrics, low density polyethylene and polyimide. Prebreakdown transient current spikes or arcs were observed, using both slow and high speed detection. These pre-ESD discharge phenomena are explained in terms of breakdown modes and defect generation on a microscopic scale. The field at which prebreakdown arcing begins was compared to the onset field for electrostatic discharge at which complete breakdown occurs for each material studied. We present evidence that these two threshold fields are the same. Thus, the important parameter to consider in design may not be the maximum field for breakdown, as much as the defect structure in the materials and the field where pre-breakdown arcing begins in a material.
\end{abstract}

Keywords-electrostatic discharge; arcing; breakdown; charging, partial discharge, dielectric materials

\section{INTRODUCTION}

Enhanced understanding of DC aging based on expanded experimental studies is of critical importance not only to understand the physics of highly disordered insulating materials (HDIM), but also for applications in spacecraft charging, high voltage DC power transmission and switching, thin film dielectrics, and semiconductor devices and sensors[13]. Recent literature on DC power cable aging calls for better models and more data on the details of electrical ageing in HDIM under DC high electric fields, especially with regards to finding a DC equivalent for $\mathrm{AC}$ partial discharge diagnostic tests[4-7].

High electric field stress phenomena associated with electrostatic discharge (ESD) were studied for low density polyethylene (LDPE) and polyimide. ESD is observed over a range of fields, extended well below the average ESD field down to a minimum field termed the onset field, $F_{\text {onset }}$. For these polymers very short duration, unsustained arcingtermed here as pre-breakdown arcing-was also observed at fields well below the average breakdown field for each material, down to a minimum field termed the onset field, $F_{\text {Pre- }}$ arcing. We propose that minimum field at which ESD begins to occur can be correlated to the initial fields for pre-breakdown arcing.

Our experimental results are explained in terms of a dualdefect thermodynamic mean field trapping model [8]. This model characterizes electrical aging in terms of recoverable defects that can be thermally annealed and irrecoverable defects with higher energies such as bond breaking. We use this model to make a statistical comparison of our arcing and ESD data.

\section{EXPERIMENTATION}

The critical field for ESD breakdown was determined by performing step-up tests of the voltage across $\sim 25 \mu \mathrm{m}$ samples and monitoring the leakage current [8]. A simple parallel-plate capacitor geometry was used, under high vacuum, to achieve field strengths of up to $590 \mathrm{MV} / \mathrm{m}[9,10]$. Current was monitored at $\sim 2 \mathrm{~Hz}$ with an ammeter and $\sim 10 \mathrm{kHz}$ with an oscilloscope. Voltage was increased incrementally at constant rate of $\Delta V_{\text {step }} \approx 20 \mathrm{~V}$ at $\Delta t_{\text {step }} \approx 3.5$ up to $30 \mathrm{kV}$ until complete breakdown occurred (see Fig. 1). Above breakdown (yellow region in Fig. 1) current increased linearly with the slope set by current limiting resisters in the circuit up through voltages where all samples have broken down (red region in Fig. 1). Prior to breakdown pre-breakdown arcing is observed with currents below breakdown currents at a given voltage (blue and yellow regions in Fig. 1).

LDPE samples from Goodfellow used had an average measured thickness of $29.7 \pm 2 \% \mu \mathrm{m}$, density of $0.92 \mathrm{~g} / \mathrm{cm}^{3}$ [11], an estimated crystallinity of 50\% [12], an estimated peak fractional mass distribution of $\sim 6 \cdot 10^{3}$ amu or $\sim 210^{3} \mathrm{C}_{2} \mathrm{H}_{4}$ mers per chain[13,14], and a relative dielectric constant of 2.26 [11]. Samples of polyimide DuPont Kapton $\mathrm{HN}^{\mathrm{TM}}$ used had a $23.9 \pm 4 \% \mu \mathrm{m}$ average measured thickness, density of $1.43 \pm 0.01$ $\mathrm{g} / \mathrm{cm}^{3}$ [15], and a relative dielectric constant of 3.5 [15]. A single mer of polyimide has an atomic composition of $\mathrm{C}_{22} \mathrm{O}_{5} \mathrm{~N}_{2} \mathrm{H}_{10}$ [15].

\section{StatisticAl ANALYSIS OF ElECTROSTATIC BREAKDOWNS}

The electrical aging of HDIM is described by many publications as depending on the Gibbs free energy, bond destruction energy, or cohesion energy associated with internal defect creation due to local and applied electric fields [4,1618]. The motion of charge carriers between defect sites either with or against an applied electric field can be modeled as a rate process similar to the hopping conductivity models of Miller and Abrahams or the standard Crine aging model which assume a single mean defect energy and density $[4,6,19,20]$. Static voltage time endurance tests of LDPE strongly suggest a need for the inclusion of multiple defect species [8]. This 


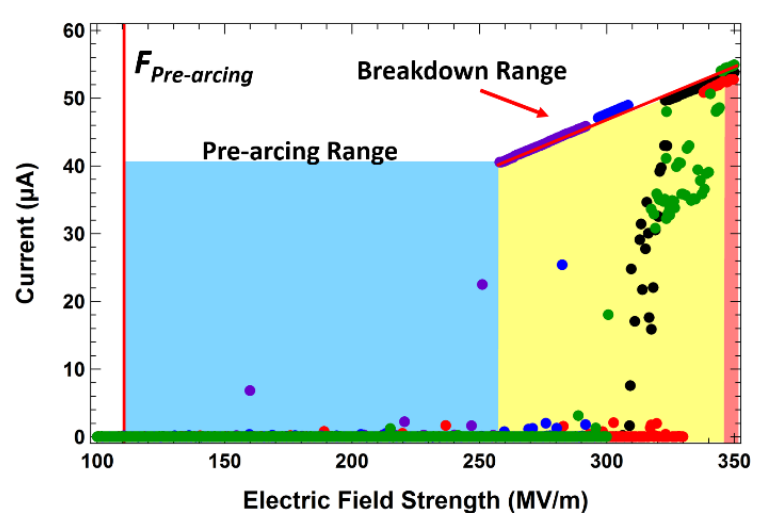

Fig. 1. Electrostatic breakdown step-up tests. Plot of five step-up tests on LDPE. These curves show complete breakdown, the intermediate region in yellow, where the IV curves join the linear ohmic trend line set by the current limiting resistors. The complete breakdown region in red is above the highest of the measured breakdowns. Pre-arcing breakdown events are seen as spikes in the current throughout the blue and yellow regions, down to $F_{\text {Pre-arcing }} \approx 110$ $\mathrm{MV} / \mathrm{m}$ for these tests.

extended model, Eq. (1), for the probability of breakdown due to two defect species $P_{T o t}$ after time $\Delta t$ at temperature $T$ subject to an applied field $F$ depends on the defect energy $\Delta G_{\text {def }}^{i}$ and density $N_{d e f}^{i}$ for each defect type $i=A, B$ [8].

$$
\begin{aligned}
& P_{d e f}^{T o t}(\Delta t, F, T)=\sum_{i=A, B} P_{d e f}^{i} \\
&=\left(\frac{2 k_{B} T}{h / \Delta t}\right) \sum_{i=A, B} \exp \left[\frac{-\Delta G_{d e f}^{i}}{k_{B} T}\right] \sinh \left[\frac{\varepsilon_{o} \varepsilon_{r} F^{2}}{2 N_{i} k_{B} T}\right]
\end{aligned}
$$

Consider two defect species, one reversible and one irreversible (Types A and B respectively). Type A defects, $\Delta G_{d e f}^{A}$ are on the order of $k_{B} T$ for relevant temperatures and can be thermally annealed at a significant rate. The repair rate of these defects is strongly temperature dependent. Physically these could arise from weak van der Waals bonds, polymer chain kinks or kink pairs [16,21]. It is expected due to strong variations in the chain structure, rigidity and crosslinking that $\Delta G_{d e f}^{A}$ will vary considerably for different polymers. Type B defects, $\Delta G_{d e f}^{B}$ are associated with broken bonds such as carbon-carbon bonds of the $\mathrm{C}_{2} \mathrm{H}_{4}$ monomer alkane single bonds along the polymer chains that act as electron traps [16, $20,22,23]$. Since $\Delta G_{\text {def }}^{B} \gg k_{B} T$ for all relevant $T$. these defects have a negligible repair rate [4]. These broken bond $(b b)$ energies have a dissociation energy of $\Delta G_{d e f}^{b b}=3.65 \mathrm{eV} / \mathrm{bond}$ [23]. We do not expect $\Delta G_{d e f}^{B}$ to vary greatly from one polymer to another because of the commonality of the carboncarbon bonds.

We performed 89 LDPE and 36 Kapton step-up tests (Section II). Fig. 3 shows the fraction of breakdowns that occur versus the breakdown electric field. The shapes of these distributions suggest that simply a mean breakdown field with some uncertainty is an inadequate description of ESD breakdown fields. Eq. 1 applied to the step-up processes leads to a formula for the cumulative probability of breakdown after $N_{\text {step }}=\frac{F D}{\Delta V}$ voltage steps of $\Delta V$ to field $F$ across a sample with thickness $D[8]$. A first order approximation to this formula is a Weibull distribution [8]

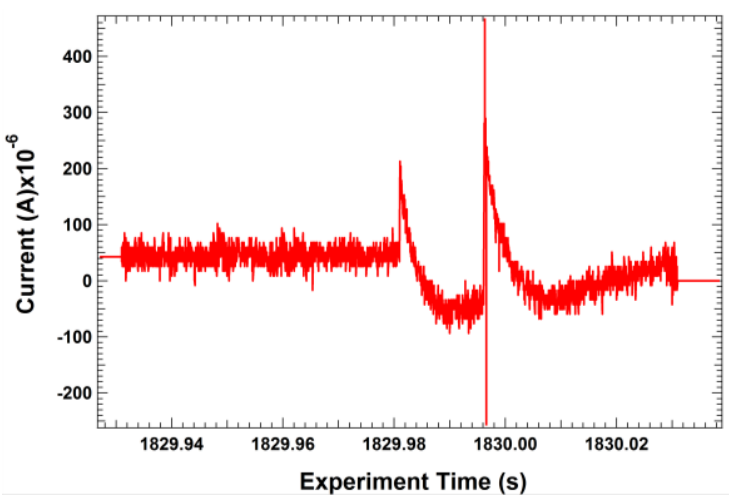

Fig. 2. Evidence of fast pre-breakdown arcing. Multiple peaks in oscilloscope measurements have been observed when large amplitude current spikes are observed in ammeter data (See Fig. 1). This suggests that these larger current spikes in ammeter data are really the current integrated over several small arcs of about the same amplitude.

$P^{W}(F)=1-\exp \left[-\left(\frac{F}{F_{d e f}^{W}}\right)^{\beta}\right]$

where $F_{\text {def }}^{W}$ approximates the field associated with the defect energy involved in breakdown. The fraction of total samples broken down versus breakdown field was fit to Eq. 2 and is shown in Fig. 3. For LDPE $F_{\text {def }}^{W}=293 \mathrm{MV} / \mathrm{m}$ and $\beta=6.96$. For Kapton $F_{d e f}^{W}=336 \mathrm{MV} / \mathrm{m}$ and $\beta=10.9$. We define the onset of breakdowns, $F_{\text {onset }}$ as $P^{W}\left(F_{\text {onset }}\right)=0.0455$. Similarly we define the field at which nearly all breakdowns have occurred, $F_{E S D}$ as $P^{W}\left(F_{E S D}\right)=0.9545$. In Figs. 3 and $4 F_{\text {onset }}$ to $F_{\text {def }}^{W}$ defines the blue region, $F_{d e f}^{W}$ to $F_{E S D}$ defines the yellow region and the red region is defined as field values above $F_{E S D}$. For LDPE $F_{\text {onset }}=189 \pm 6 \mathrm{MV} / \mathrm{m}$ while $F_{E S D}=345 \pm 17 \mathrm{MV} / \mathrm{m}$. For Kapton $F_{\text {onset }}=253 \pm 8 \mathrm{MV} / \mathrm{m}$ while $F_{E S D}=373 \pm 11$ $\mathrm{MV} / \mathrm{m}$. In Fig. 3 we see similar high field behavior which would be expected if Type B defects are roughly the same for each material. The blue regions in Fig. 3 associated with $F_{\text {onset }}$ differ significantly corresponding to large differences in Type A defects. For Kapton, the sturdier of the two materials, we see that $F_{\text {onset }}$ is comparatively higher than for LDPE.

\section{Statistical ANalysis of PRE-ARCING}

A statistical analysis has been conducted of the common, short-duration, recoverable discharge events or pre-arcs observed during ESD test before complete breakdown occurs. The data discussed in this section are the results of the same step-up voltage tests discussed in Section III.

Typical threshold amplitudes for the smallest arcs observed above background noise in the ammeter data were $0.09 \mu \mathrm{A} \mathrm{s}$ for LDPE and $0.07 \mu \mathrm{A}$ for polyimide. The pre-arcs measured with slow ammeters (see Fig. 1) are more frequent and of higher current amplitude at higher applied fields. This suggests that a correction for the measured arc rate is needed to correct for multiple short duration arcing events occurring within a single $\sim 0.5 \mathrm{~s}$ data acquisition interval of the ammeters used in these experiments and are integrated and averaged by the ammeter. Typical discharge pulses observed with fast oscilloscopes are $<5 \mu$ s duration (see Fig. 2). The estimated ammeter amplitude of a single arc is $0.15 \pm 0.05 \mu \mathrm{A}$. Shapes of the distributions of the arc rates (see Fig. 4) are largely 

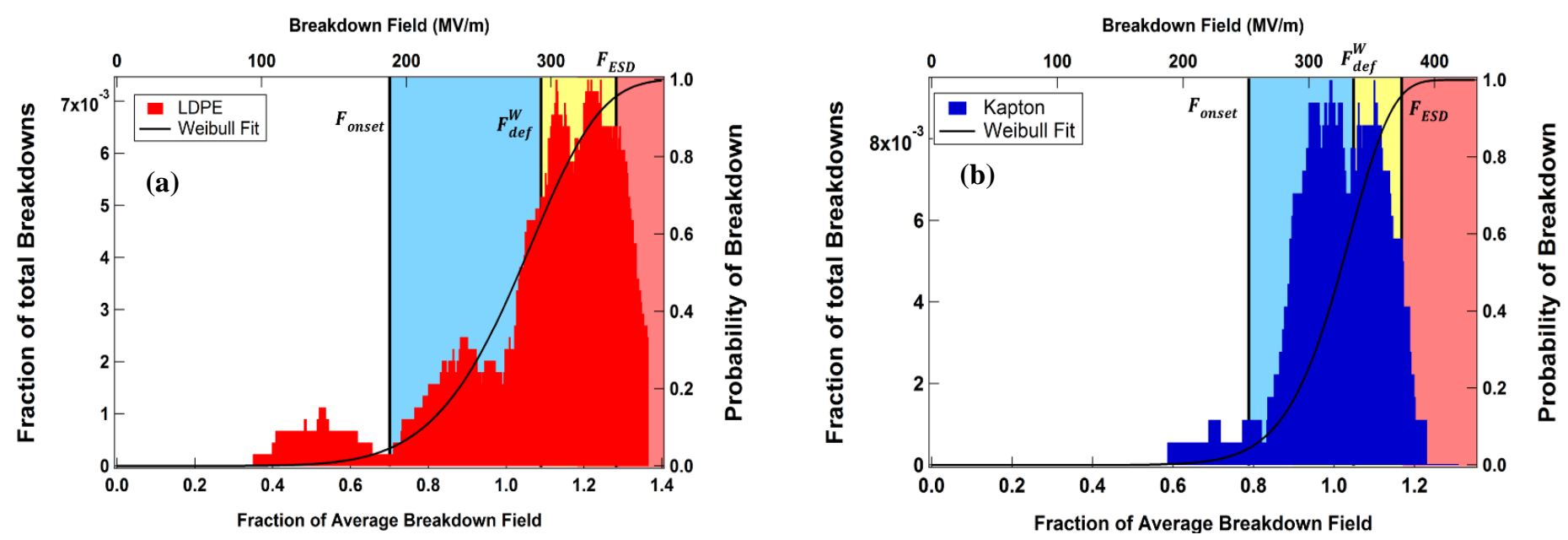

Figure 3. Histograms of the fraction of total breakdowns vs breakdown electric field compared to the probability of breakdown at those fields given by Weibull distributions (black curves) for (a) 89 LDPE and (b) 36 Kapton step-up breakdown tests.
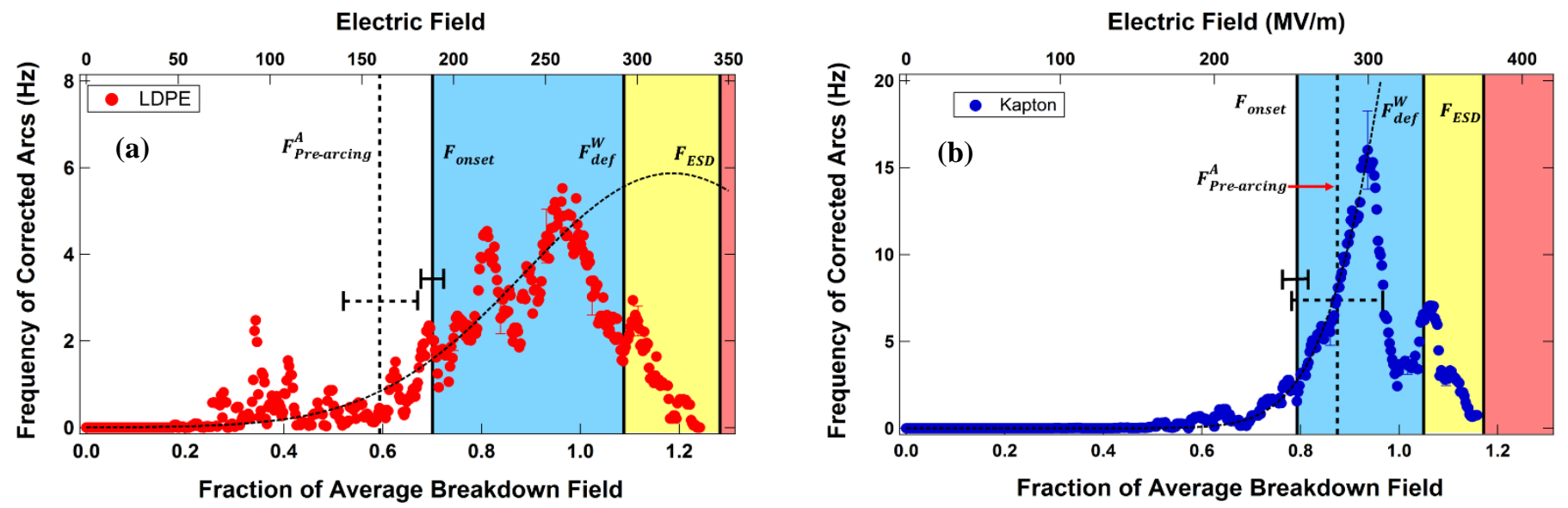

Figure 4. Histograms of the frequency of short duration, recoverable breakdown events or "pre-arcing" observed during 89 LDPE and 36 Kapton step-up breakdown tests for (a) LDPE (89 tests) and (b) polyimide (36 tests). Frequencies have been corrected as described in the text. Black dashed curves are Gaussian fits, based on Eq. (3). Error bars on $F_{\text {Pre-arcing }}^{A}$ and $F_{\text {onset }}$ are shown.

insensitive to the choice of the threshold values. The arcing rates measured with the ammeter were corrected for these multiple arcs per acquisition time by estimating the number of single arcs in higher current events as the measured current divided by the average single arc current

Figure 4 shows a histogram of number of corrected prebreakdown arcing events versus applied field divided by the average breakdown field for both LDPE and polyimide step-up tests. These frequency data are fit with a field-dependent Gaussian distribution

$$
f(F)=\frac{f_{0}}{\sqrt{2 \pi} \Delta F} \exp \left[-\left(\frac{(F-\bar{F})^{2}}{2 \Delta F^{2}}\right)\right]
$$

centered at $\bar{F} \approx F_{D e f}^{B}$, the peak in the data, with a width $\Delta F=\frac{1}{2}\left(\bar{F}-F_{P r e-a r c}^{A}\right)$ and normalized to $f_{0}$. The frequencies have been corrected to reflect only $\sim 0.5 \mathrm{~s}$ ammeter data collection interval per $3.5 \mathrm{~s}$ at each voltage. $F_{\text {Pre-arcing }}^{A}=160$ $\pm 20 \mathrm{MV} / \mathrm{m}$ for LDPE and $F_{\text {Pre-arcing }}^{A}=280 \pm 30 \mathrm{MV} / \mathrm{m}$ for polyimide. For LDPE $f_{0}=1200 \pm 400 \mathrm{MV} / \mathrm{m} \cdot \mathrm{s}, \Delta F=80 \pm 10$ $\mathrm{MV} / \mathrm{m}$ and $\bar{F}=310 \pm 30 \mathrm{MV} / \mathrm{m}$. For Kapton $f_{0}=7000 \pm 3000$ $\mathrm{MV} / \mathrm{m} \cdot \mathrm{s}, \Delta F=53 \pm 3 \mathrm{MV} / \mathrm{m}$ and $\bar{F}=384 \pm 17$.
The Gaussian fit is a first-order approximation to the arc rate assuming a random distribution of pre-arcing events with average breakdown, but does not account for removal of specimens from the sample population after breakdown; a more complete theory (like that used for the ESD analysis above) would use the Weibull function to account for these failures and provide a better model near and above the distribution peak. However, to identify the approximate peak and upper bound of the distribution of pre-arcs, and most importantly the lower bound of the pre-arc distribution, this model is sufficient. It has been suggested that the observed Gaussian distribution is related to the flexibility of polyethylene chains in the amorphous regions of LDPE [8]. A similar Gaussian distribution of the mean radius of random polymer coils is predicted from random walk models of chain kink density [24, 25]. This can be related to the entropic contribution to elastic strain energy for basic theories of rubber elasticity $[21,26]$. The critical field associated with one defect per activation volume is given by [21]

$$
F_{d e f}=\left[\left(2 / \varepsilon_{0} \varepsilon_{r}\right) N_{d e f} \Delta G_{d e f}\right]^{\frac{1}{2}} \text {. }
$$


Using values for LDPE, $\Delta G_{\text {def }}^{B}=3.65[23]$ and $N_{d e f}^{A}=1.75^{*} 10^{18}$ $\mathrm{cm}^{-3}$ from fitting static voltage time endurance data [8] $F_{d e f}=320 \mathrm{MV} / \mathrm{m}=\bar{F}$ within our uncertainty.

Let us now directly compare $F_{\text {onset }}$ to $F_{\text {Pre-arcing. A }}^{A}$ cursory observation of Figs. 3 and 4 suggests that for both LDPE and Kapton ESD breakdown can begin to happen at about the same field as pre-arcing is observed in samples that haven't broken down yet. To make a quantitative comparison we assumed a Gaussian probability for pre-arcing up to where the frequency starts to decrease and a Weibull distribution probability for breakdown. Now comparing the fields at which we have a $\sim 5 \%$ probability of observing either arcing $\left(F_{\text {Pre-arcing }}^{A}\right)$ or breakdown $\left(F_{\text {onset }}\right)$ we see that for LDPE $F_{\text {Pre-arcing }}^{A}=160 \pm 20 \mathrm{MV} / \mathrm{m} \approx F_{\text {onset }}=189 \pm 6 \mathrm{MV} / \mathrm{m}$ and for Kapton $F_{\text {Pre-arcing }}^{A}=280 \pm 30 \mathrm{MV} / \mathrm{m}=F_{\text {onset }}=253 \pm 8 \mathrm{MV} / \mathrm{m}$ within the uncertainty. Transient pre-arcs are consistent with a percolation-like model for recoverable (Type A) defects resulting in a finite probability of breakdown well below more probable breakdown fields [8].

\section{CONClusions}

This study of step-up measurements of LDPE and Kapton measurements indicates that the field for the onset of catastrophic ESD breakdown can be estimated as the same field where pre-breakdown arcing begins. Our dual-defect model suggests possible physical origins for the behavior observed in terms of recoverable and irrecoverable defects. Measurements of $F_{\text {Pre-arcing are considerably easier than }}^{A}$ measurements of $F_{\text {onset }}$ since many pre-arcs are generally observed in a single step-up test. Using $F_{\text {Pre-arcing as an }}^{A}$ estimate of $F_{\text {onset }}$ has the potential to expedite diagnostics of insulating materials in practical applications.

\section{ACKNOWLEDGMENT}

Research was supported by funding from NASA Goddard Space Flight Center, a USU Blood Fellowship (Andersen), a NASA Graduate Research Fellowship (Andersen), and a Senior Research Fellowship from the Air Force Research Laboratory through the National Research Council (Dennison). We gratefully acknowledge useful discussions with Alec Sim and help with instrumentation and experiments from Dan Arnfield, Anthony Thomas, Jeri Brunson, Ryan Hoffmann, Justin Dekany, and Matthew Stromo,

\section{REFERENCES}

[1] H. B. Garrett, and A. C. Whittlesey, "Spacecraft charging, an update," Plasma Science, IEEE Transactions on, vol. 28, no. 6, pp. 2017-2028, 2000.

[2] K. L. Bedingfield, R. D. Leach, and M. B. Alexander, Spacecraft system failures and anomalies attributed to the natural space environment: National Aeronautics and Space Administration, Marshall Space Flight Center, 1996.

[3] G. Teyssedre, and C. Laurent, "Advances in high-field insulating polymeric materials over the past 50 years," Electrical Insulation Magazine, IEEE, vol. 29, no. 5, pp. 26-36, 2013.

[4] J. P. Crine, "On the interpretation of some electrical aging and relaxation phenomena in solid dielectrics," Dielectrics and Electrical Insulation, IEEE Transactions on, vol. 12, no. 6, pp. 1089-1107, 2005.
[5] T. T. N. Vu, G. Teyssedre, B. Vissouvanadin et al., "Electric field profile measurement and modeling in multi-dielectrics for HVDC application." pp. 413-416.

[6] P. Trnka, M. Sirucek, M. Svoboda et al., "Condition-based maintenance of high-voltage machines-a practical application to electrical insulation," Electrical Insulation Magazine, IEEE, vol. 30, no. 1, pp. 32-38, 2014.

[7] T. Czaszejko, "High-voltage testing fundamentals: a cable testing perspective," Electrical Insulation Magazine, IEEE, vol. 30, no. 1, pp. $7-$ 13, 2014.

[8] A. Andersen, J. R. Dennison, A. M. Sim et al., "Electrostatic Discharge and Endurance Time Measurements of Spacecraft Materials: A DefectDriven Dynamic Model," in 13th Spacecraft Charging Technology Conference, Pasadena, CA, 2014.

[9] ASTM D 149-97a, "Standard Test Method for Dielectric Breakdown Voltage and Dielectric Strength of Solid Electrical Insulating Materials at Commercial Power Frequencies," (American Society for Testing and Materials, 100 Barr Harbor Drive, West Conshohocken, PA 19428, 2004).

[10] ASTM D3755 - 14, "Standard Test Method for Dielectric Breakdown Voltage and Dielectric Strength of Solid Electrical Insulating Materials Under Direct-Voltage Stress," (American Society for Testing and Materials, 100 Barr Harbor Drive, West Conshohocken, PA 19428, 2014).

[11] "Material Information-Polyethylene Low Density LDPE," .Goodfellow, Devon, PA, January 20, 2006.

[12] H. J. Wintle, "Conduction Processes in Polymers," Engineering Dielectrics-Volume IIA: Electrical Properties of Solid Insulating Materials: Molecular Structure and Electrical Behavior, R. Bartnikas, ed., Philadelphia, PA: American Society for Testing and Materials, 1983.

[13] J. Brunson, "Measurement of charge decay time and resistivity of spacecraft insulators using charge storage method and application to theoretical modeling of charging behavior of insulators," Physics. Vol. PhD, Utah State University, Logan, UT, pp. 215, 2009.

[14] A. Peacock, Handbook of polyethylene: structures: properties, and applications: CRC Press, 2000.

[15] "Dupont Kapton HN Polyimide Film," Dupont Technical Bulletin H38479, GS-96-7, Circleville, OH, 2011.

[16] K. C. Kao, Dielectric phenomena in solids: Academic press, 2004.

[17] T. Lewis, "Polyethylene under electrical stress," Dielectrics and Electrical Insulation, IEEE Transactions on, vol. 9, no. 5, pp. 717-729, 2002.

[18] G. Montanari, C. Laurent, G. Teyssedre et al., "From LDPE to XLPE: investigating the change of electrical properties. Part I. space charge, conduction and lifetime," Dielectrics and Electrical Insulation, IEEE Transactions on, vol. 12, no. 3, pp. 438-446, 2005.

[19] A. Miller, and E. Abrahams, "Impurity Conduction at Low Concentrations," Physical Review, vol. 120, no. 3, pp. 745-755, 1960.

[20] J.-P. Crine, J.-L. Parpal, and C. Dang, "A new approach to the electric aging of dielectrics." pp. 161-167.

[21] J. C. Anderson, K. D. Leaver, R. D. Rawlings et al., Materials science for engineers: CRC Press, 2004.

[22] T. Lewis, J. Llewellyn, M. Van der Sluijs et al., "A new model for electrical ageing and breakdown in dielectrics." pp. 220-224.

[23] P. Phillips, "Morphology and molecular structure of polymers and their dielectric behavior," Engineering Dielectrics Volume Iia Electrical Properties of Solid Insulating Materials: Molecular Structure and Electrical Behavior, pp. 119, 1983.

[24] H. Cho, Y. C. Kim, S. O. Kim et al., "Persistence length calculation from light scattering and intrinsic viscosity of dilute semiflexible polyimide solutions with different degree of imidization," KoreaAustralia Rheology Journal, vol. 12, no. 1,

[25] R. Zallen, The Physics of Amorphous Solids, New York, NY: Wiley, 1983.

[26] K. SHINYAMA, and S. FUJITA, "Mechanical and Dielectric Breakdown Properties Eco-Friendly Dielectric Materials," 2006. 